Supporting Information

\title{
Rapid differential diagnosis of seven human respiratory coronavirus based on centrifugal microfluidic nucleic acid assay
}

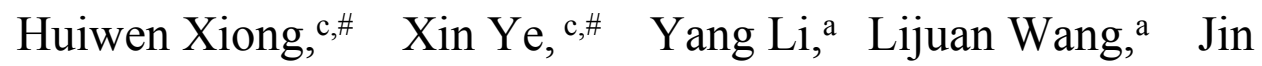 \\ Zhang, ${ }^{\mathrm{b}, *}$ Xueen Fang, ${ }^{\mathrm{c}, *}$ and Jilie Kong ${ }^{\mathrm{c}, *}$ \\ ${ }^{\text {a }}$ Shanghai Suxin Biotechnology Co. Ltd, and IgeneTec Diagnostic products Co. Ltd. Shanghai \\ 201318, P. R. China \\ ${ }^{\mathrm{b}}$ Qingdao International Travel Healthcare Center, Qingdao Customs, Qingdao 266071, \\ P. R. China \\ ${ }^{\mathrm{c}}$ Department of Chemistry and Institutes of Biomedical Sciences, Fudan University, Shanghai \\ 200433, P. R. China
}

\section{Corresponding Author}

*Xueen Fang, fxech@,fudan.edu.cn;

*Jilie Kong, jlkong@fudan.edu.cn

*Jin Zhang, zhjoohyn@hotmail.com

\section{Co-first Author}

${ }^{\#}$ H. X. and X.Y. contributed equally to this work.

\section{Author Contributions}

All authors have given approval to the final version of the manuscript. 


\section{Table of Content}

\section{Experimental Section}

Figure. S1 (A) Photograph of LAMP-microfluidic chip under $480 \mathrm{~nm}$ emission light;

(B) Photograph of LAMP-microfluidic chip under sunlight.

Figure. S2 The primer set selection results for each virus. (A) The primer sets for

HCoV-229E; (B) The primer sets for HCoV-OC43; (C) The primer sets for HCoVNL63; (D) The primer sets for HCoV-HKU1; (E) The primer sets for SARS-CoV; (F) The primer sets for MERS-CoV. (G) The primer sets for SARS-CoV-2 targeting N; (H) The primer sets for SARS-CoV-2 targeting ORF1ab, respectively. X axis represents time ( $\min ), Y$ axis represents relative fluorescence intensity (a. u.) in this figure.

Table S1. Primer sets for HCoV-229E microfluidic LAMP assay.

Table S2. Primer sets for HCoV-OC43 microfluidic LAMP assay.

Table S3. Primer sets for HCoV-NL63 microfluidic LAMP assay.

Table S4. Primer sets for HCoV-HKU1 microfluidic LAMP assay.

Table S5. Primer sets for SARS-CoV microfluidic LAMP assay.

Table S6. Primer sets for MERS-CoV microfluidic LAMP assay.

Table S7. Primer sets for SARS-CoV-2 targeting ORF1ab microfluidic LAMP assay.

Table S8. Primer sets for SARS-CoV-2 targeting N microfluidic LAMP assay. 


\section{Materials and methods}

\section{Reagents and instruments}

All primers in the study were synthesized and purified by BIOLIGO (Shanghai, China). Trehalose was obtained from Sigma. Total nucleic acid extraction kit (No. 20170099) and buffer with enzymes and fluorescent dyes were purchased from Shanghai Suchuang Diagnostics Co., Ltd. The fluorescence intensity was recorded by MA2000Plus (Shanghai Suxin Biotechnology).

\section{Design of LAMP primers}

We utilized Primer Explorer V5 (http://primerexplorer.jp/e/index.html. Eiken Chemicals Corporation, Tokyo, Japan) to design the primers. Four sets of primers each were designed for the initial selection of HCoV-229E, HCoV-OC43, HCoV-NL63, HCoV-HKU1, SARS-CoV, MERS-CoV, SARS-CoV-2 targeting N sequence and SARS-CoV-2 targeting ORF1ab sequence were designed initially for selection.

\section{Clinical samples and nucleic acid extraction}

All clinical throat swab samples were provided by Qingdao Administration of Entry \& Exit Inspection and Quarantine Bureau, patients involved were infected by a single pathogen: HCoV-229E ( $\mathrm{n}=3$ ), HCoV-OC43 ( $\mathrm{n}=3)$, HCoV-NL63 (n=3), HCoVHKU1 ( $n=3)$, MERS-CoV ( $n=3)$ and SARS-CoV-2 $(n=12)$. SARS-CoV samples were not included for there had not been any cases in many years, so a plasmid carrying its sequences was used as an alternative. Informed consent was received from all subjects involved. All unprocessed samples were stored at $-80{ }^{\circ} \mathrm{C}$.

Nucleic acids, including DNA and RNA, were extracted from viral stocks using a 
commercial kit following the manufacturers' instructions. The extracted nucleic acids were then divided into two sets. One set was reverse-transcribed to cDNA (except for SARS-CoV; plasmid samples) and stored at $-20^{\circ} \mathrm{C}$. The second set was stored at $-80{ }^{\circ} \mathrm{C}$ for further use. Plasmids carrying the highly conserved gene sequences of HCoV-229E, HCoV-OC43, HCoV-NL63, HCoV-HKU1, SARS-CoV, MERS-CoV and SARS-CoV2 were purchased from Shanghai BIOLIGO.

\section{LAMP assays}

The total volume of each LAMP reaction system in each reaction hole was $5 \mu \mathrm{L}$. The outer primer (F3, B3; $0.2 \mu \mathrm{M})$, inner primer (FIP, BIP; $1.6 \mu \mathrm{M})$, loop primer (LF, $\mathrm{LB} ; 0.8 \mu \mathrm{M}), 0.26 \mu \mathrm{L}$ trehalose, $0.98 \mu \mathrm{L}$ ultrapure water were preloaded in the reaction chambers and dried for storage. $12 \mu \mathrm{L}$ nucleic acids template and $60 \mu \mathrm{L}$ amplification buffer were added to the sample adding chamber, which was then sealed with film. After centrifugation, liquids in the sample adding chamber were pumped into reaction chambers. LAMP reactions in HCoV-229E, HCoV-OC43, HCoV-NL63, HCoV-HKU1, SARS-CoV, MERS-CoV and SARS-CoV-2 were processed at $65{ }^{\circ} \mathrm{C}$ for $40 \mathrm{~min}$. Fluorescence of SYTO-9 provided real-time output.

\section{Optimizing the designed primers of a LAMP-based microfluidic platform}

Four sets of primers including loop primers (LF/LB) were designed for each coronavirus (Table S1-S8). In consideration of short cycles to present positive signals, we chose primer set No. 2 for HCoV-229E, No. 3 for HCoV-OC43, No. 4 for HCoVNL63, No. 3 for HCoV-HKU1, No. 4 for SARS-CoV, No. 1 for MERS-CoV, No. 2 targeting N and No. 1 targeting ORF1ab sequences for SARS-CoV-2 (Figure S2). 

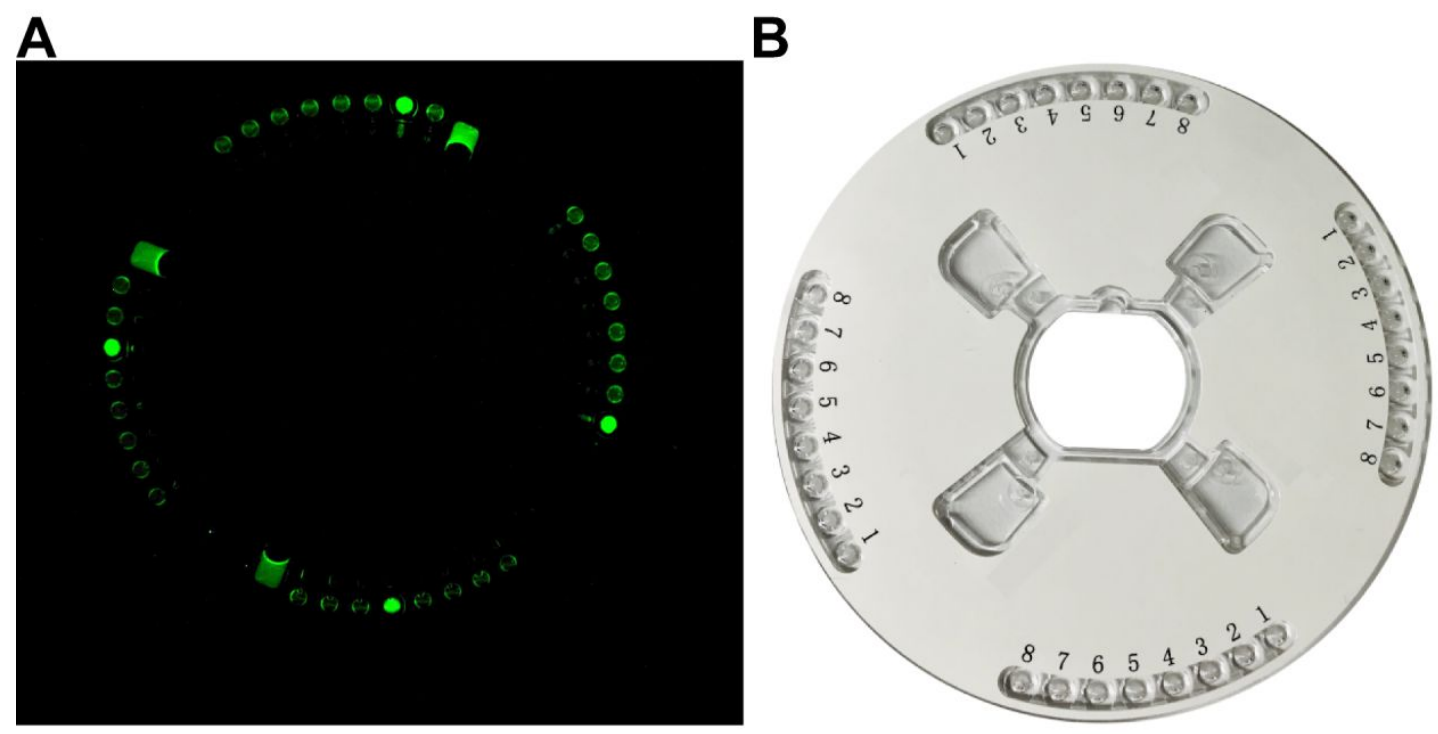

Figure. S1 (A) Photograph of LAMP-microfluidic chip under $480 \mathrm{~nm}$ emission light;

(B) Photograph of LAMP-microfluidic chip under sunlight.
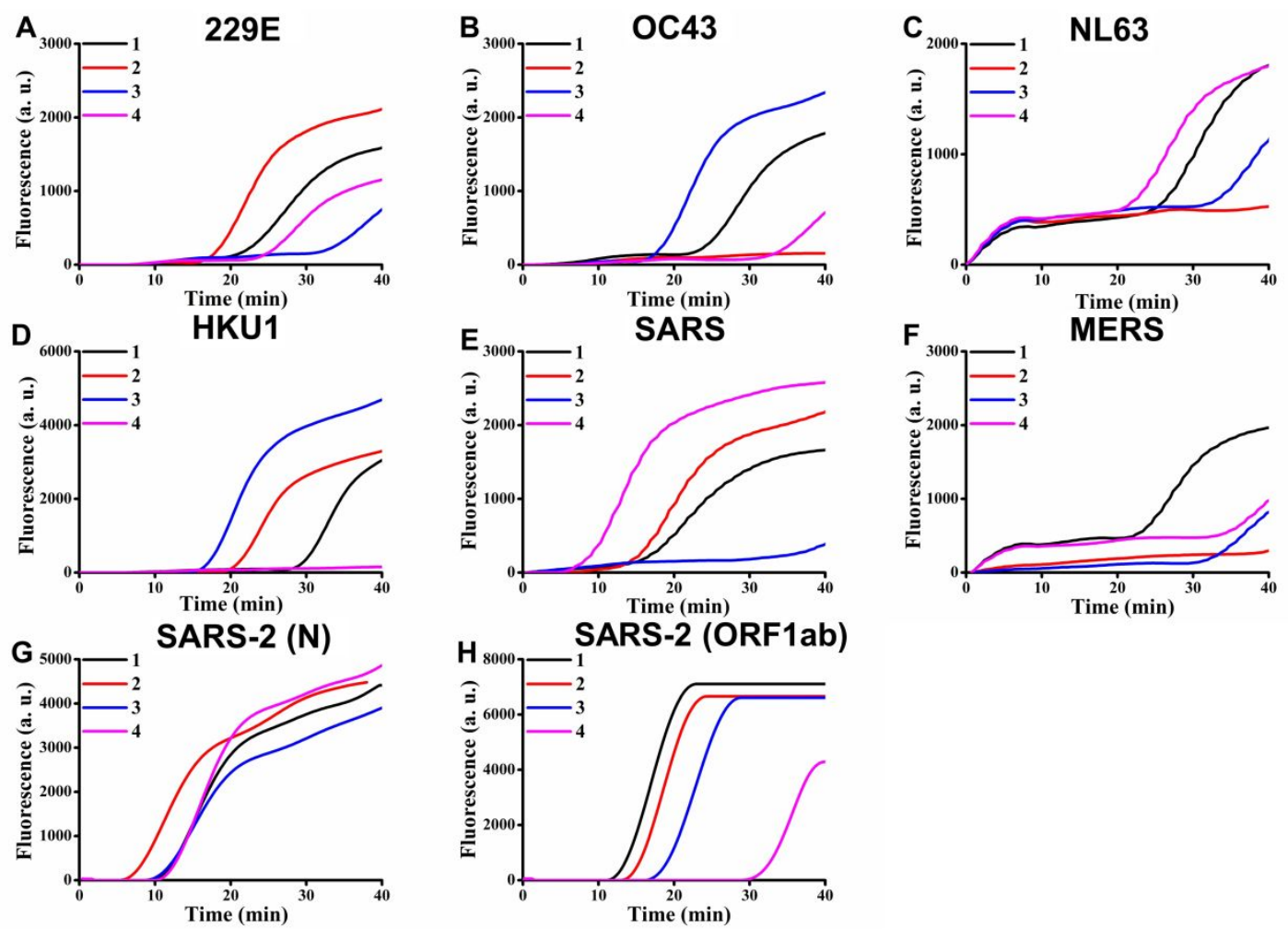

Figure. S2 The primer set selection results for each virus. (A) The primer sets for 
HCoV-229E; (B) The primer sets for HCoV-OC43; (C) The primer sets for HCoVNL63; (D) The primer sets for HCoV-HKU1; (E) The primer sets for SARS-CoV; (F) The primer sets for MERS-CoV. (G) The primer sets for SARS-CoV-2 targeting N; (H) The primer sets for SARS-CoV-2 targeting ORF1ab, respectively. $\mathrm{X}$ axis represents time (min), Y axis represents relative fluorescence intensity (a. u.) in this figure.

Table S1. Primer sets for HCoV-229E microfluidic LAMP assay

\begin{tabular}{|l|l|}
\hline Project & Primer sequence $\left(5^{\prime}\right.$ '-3') \\
\hline F3 & GCATCTGAACCACAACGT \\
\hline B3 & GTGACAAATCCACCCGTT \\
\hline FIP & $\begin{array}{l}\text { CGAGGTATCACCTTCCAAGGTTCCTTATTCTCTTTATAGCCCTTT } \\
\text { G }\end{array}$ \\
\hline BIP & $\begin{array}{l}\text { TTGGTACCCATCAACAAGAAAGACCCCTTTCTAGTTCTGAAAC } \\
\text { G }\end{array}$ \\
\hline LB & AGCTTATAGGCTATTGGAATGTTCA \\
\hline Target & $\begin{array}{l}\text { GCATCTGAACCACAACGTGTGACAAATCCACCCGTTCGAGGTA } \\
\text { CCCATCAACAAGAAAGACCCCTTTCTAGTTCTGAAACG }\end{array}$ \\
\hline
\end{tabular}

Table S2. Primer sets for HCoV-OC43 microfluidic LAMP assay

\begin{tabular}{|c|c|}
\hline Project & Primer sequence $\left(5^{\prime}-3^{\prime}\right)$ \\
\hline F3 & TCAATACCCCGGCTGACA \\
\hline B3 & GGCTCTACTACGCGATCCT \\
\hline FIP & GTACCGTGCCAGGCGGAAACTTGTCGATCGGGACCCAA \\
\hline BIP & TTGAAGGCTCAGGAAGGTCTGCGAGGCTCTGCTGGATGTG \\
\hline $\mathrm{LF}$ & TCGGAATAGCCTCATCGCTAC \\
\hline target & $\begin{array}{l}\text { GCTGATGTCAATACCCCGGCTGACATTGTCGATCGGGACCCAA } \\
\text { GTAGCGATGAGGCTATTCCGACTAGGTTTCCGCCTGGCACGGT } \\
\text { ACTCCCTCAGGGTTACTATATTGAAGGCTCAGGAAGGTCTGCT } \\
\text { CCTAATTCCAGATCTACTTCGCGCACATCCAGCAGAGCCTCTAG } \\
\text { TGCAGGATCGCGTAGTAGAGCCAATTCTGGCAATAGAACCCCT }\end{array}$ \\
\hline
\end{tabular}




\begin{tabular}{|l|l|}
\hline ACCTCTGGTGTAACACCTGACATGGCTGATCAAATTGCTAGTCT \\
TGTTCTGGCAAAACTTGGCAAGGATGCCACTAAACCTCAGCAA \\
GTAACTAAGCATACTGCCAAAGAAGTCAGACAGAAAATTTTGA \\
ATAAGCCCCGCCAGAAGAGGAGCCCCAATAAACAATGCACTGT \\
TCAGCAGTGTT
\end{tabular}

\section{Table S3. Primer sets for HCoV-NL63 microfluidic LAMP assay}

\begin{tabular}{|c|c|}
\hline Project & Primer sequence $\left(5^{\prime}-3^{\prime}\right)$ \\
\hline F3 & TGACTCTTCATGGCACATT \\
\hline B3 & CCAGTAATGGAATTACCTTCAGA \\
\hline FIP & CGACGGTTGAGAAACAAATAGTCTTAAAGAGTGGCACTTGTCC \\
\hline BIP & $\begin{array}{l}\text { TGCCTGGTAGTTGTAATTTTCCACCAAGTAACATACAAAGCAC } \\
\text { C }\end{array}$ \\
\hline LB & AAGCTACCTGGCATTACACTTCT \\
\hline target & $\begin{array}{l}\text { CCGCGTTAAGAGTGGTTCACCAGGTGACTCTTCATGGCACATTT } \\
\text { ATTTAAAGAGTGGCACTTGTCCATTTTCTTTTTCTAAGTTAAAT } \\
\text { AATTTTCAAAAGTTTAAGACTATTTGTTTCTCAACCGTCGCAGT } \\
\text { GCCTGGTAGTTGTAATTTTCCACTTGAAGCTACCTGGCATTACA } \\
\text { CTTCTTATACTATTGTTGGTGCTTTGTATGTTACTTGGTCTGAAG } \\
\text { GTAATTCCATTACTGGTGTACCTTATCCTGTCTCTGGTATTCGT } \\
\text { GAGTTTAGTAATTTAGTTTTAAATAATTGTACCAAATATAATAT } \\
\text { TTATGATTATGTTGGTACTGGAATTATACGTTCTTCAAACCAGT } \\
\text { CACTTGCTGGTGGTATTACATATGTTTCTAACTCTGGTAATTTA } \\
\text { CTTGGTTTTAAAAATGTTTCCACTGGTAACATTTTTATTGTGAC } \\
\text { ACCATGTAACCAACCAGACCAAGTAGCTGTTTATCAACAAAGC } \\
\text { ATTATTGGTGCCATGACTGCTGTTAATGAGTCTAGATATGGCTT } \\
\text { GCAAAACTTACTACAGTTACCTAACTTTTATTATGTTAGTAATG } \\
\text { GTAGTAACAATTGCACTACGGCCGTTATGACTTATTC }\end{array}$ \\
\hline
\end{tabular}

Table S4. Primer sets for HCoV-HKU1 microfluidic LAMP assay

\begin{tabular}{|l|l|}
\hline Project & Primer sequence $\left(5^{\prime}-3\right.$ ') \\
\hline F3 & CTGACCAATCTGAACGAAAT \\
\hline
\end{tabular}




\begin{tabular}{|c|c|}
\hline B3 & GGGTACTCCGAAAGCAATG \\
\hline FIP & $\begin{array}{l}\text { AGTATTTCCTTGTGGTTGAGTAGACCCTTTAATAGAGGCAGAA } \\
\text { AAACC }\end{array}$ \\
\hline BIP & ATCCCACATTATTCCTGGTTCTCCGGAACTCCTTGACCATCTG \\
\hline LB & GGGATCACTCAATTTCA \\
\hline target & $\begin{array}{l}\text { ATGTCTTATACTCCCGGTCATTATGCTGGAAGTAGAAGCTCCTC } \\
\text { TGGAAATCGTTCAGGAATCCTCAAGAAAACTTCTTGGGCTGAC } \\
\text { CAATCTGAACGAAATTACCAAACCTTTAATAGAGGCAGAAAAA } \\
\text { CCCAACCTAAATTCACTGTGTCTACTCAACCACAAGGAAATAC } \\
\text { TATCCCACATTATTCCTGGTTCTCCGGGATCACTCAATTTCAAA } \\
\text { AAGGTAGAGACTTTAAATTTTCAGATGGTCAAGGAGTTCCCAT } \\
\text { TGCTTTCGGAGTACCCCCTTCTGAAGCAAAAGGATATTGGTAT } \\
\text { AGACACAGCCGGCGTTCTTTTAAAACAGCTGATGGTCAACAAA } \\
\text { A }\end{array}$ \\
\hline
\end{tabular}

Table S5. Primer sets for SARS-CoV microfluidic LAMP assay

\begin{tabular}{|c|c|}
\hline Project & Primer sequence $\left(5^{\prime}-3^{\prime}\right)$ \\
\hline F3 & GTTTAATGGCACTTCTTGGTT \\
\hline B3 & TCGCCAAGATCAACATCTG \\
\hline FIP & $\begin{array}{l}\text { AACGACATCACAATTTCCTGAGACAAGAGGAACTTCTTTTCTCC } \\
\text { AC }\end{array}$ \\
\hline BIP & ATTGGCATCATTAACAACACAGTTTTTGAAGTACTTGTCCAGCT \\
\hline LB & ATGATCCTCTGCAACCTGAGC \\
\hline target & $\begin{array}{l}\text { GTCACGTATGTGCCATCCCAGGAGAGGAACTTCACCACAGCGC } \\
\text { CAGCAATTTGTCATGAAGGCAAAGCATACTTCCCTCGTGAAGG } \\
\text { TGTTTTTGTGTTTAATGGCACTTCTTGGTTTATTACACAGAGGA } \\
\text { ACTTCTTTTCTCCACAAATAATTACTACAGACAATACATTTGTC } \\
\text { TCAGGAAATTGTGATGTCGTTATTGGCATCATTAACAACACAG } \\
\text { TTTATGATCCTCTGCAACCTGAGCTTGACTCATTCAAAGAAGAG } \\
\text { CTGGACAAGTACTTCAAAAATCATACATCACCAGATGTTGATC } \\
\text { TTGGCGACATTTCAGGCATTAACGCTTCTGTCGTCAACATTCAA }\end{array}$ \\
\hline
\end{tabular}




AAAGAAATTGACCGCCTCAATGAGGTCGCTAAAAATTTAAATG
AATCACTCATTGACCTTCAAGAATTGGGAAAATATGAGCAATA
TATTAAATGGCCTTGGTATGTTTGGCTCGGCTTCATTGCTGGAC
TAATTGCCATCGTCATGGTTACAATCTTGCTTTGTTGCATGACT
AGTTGTTGCAGTTGCCTCAAGGGTGCATGCTCTTGTGGTTCTTG
CTGCAAGTTTGATGAGGATGACTCTGAGCCAGTTCTCAAGGGT
GTCAAATTACAT

Table S6. Primer sets for MERS-CoV microfluidic LAMP assay

\begin{tabular}{|c|c|}
\hline Project & Primer sequence $\left(5^{\prime}-3^{\prime}\right)$ \\
\hline F3 & CTACTATGGGTCCCGTGT \\
\hline B3 & GAAGCCTGTCATACATTGC \\
\hline FIP & $\begin{array}{l}\text { TTCTTGGACAAAGGGTAACATAGTTGAGGCTAATCCATTAGTC } \\
\text { TCT }\end{array}$ \\
\hline BIP & $\begin{array}{l}\text { ACCGTAGTATGTGCTATAACACTCTACACATAATCTAGTAGCC } \\
\text { GTAAG }\end{array}$ \\
\hline $\mathrm{LF}$ & CGTTTTCCATATGTCCAAAG \\
\hline target & $\begin{array}{l}\text { CTCCTTAAACGGCAATGTTTCTACTGTTTTCGTGCCTGCAACGC } \\
\text { GCGATTCAGTTCCTCTTCACATAATCGCCCCGAGCTCGCTTATC } \\
\text { GTTTAAGCAGCTCTGCGCTACTATGGGTCCCGTGTAGAGGCTA } \\
\text { ATCCATTAGTCTCTCTTTGGACATATGGAAAACGAACTATGTTA } \\
\text { CCCTTTGTCCAAGAACGAATAGGGTTGTTCATAGTAAACTTTTT } \\
\text { CATTTTTACCGTAGTATGTGCTATAACACTCTTGGTGTGTATGG } \\
\text { CTTTCCTTACGGCTACTAGATTATGTGTGCAATGTATGACAGGC } \\
\text { TTCAATACCCTGTTAGTTCAGCCCGCATTATACTTGTATAATAC } \\
\text { TGGACGTTC }\end{array}$ \\
\hline
\end{tabular}

Table S7. Primer sets targeting ORF1ab for SARS-CoV-2 microfluidic LAMP assay

\begin{tabular}{|l|l|}
\hline Project & Primer sequence (5' -3 ') \\
\hline O-F3 & ACTTAAAAACACAGTCTGTACC \\
\hline O-B3 & TCAAAAGCCCTGTATACGA \\
\hline O-FIP & TGACTGAAGCATGGGTTCGCGTCTGCGGTATGTGGAAAG \\
\hline
\end{tabular}




\begin{tabular}{|l|l|}
\hline O-BIP & GCTGATGCACAATCGTTTTTAAACGCATCAGTACTAGTGCCTGT \\
\hline O-LF & AGTTGATCACAACTACAGCCATAAC \\
\hline O-LB & GGTTTGCGGTGTAAGTGCA \\
& $\begin{array}{l}\text { AAGGTTACACTTAAAAACACAGTCTGTACCGTCTGCGGTATGTGGA } \\
\text { AGTGCAGCCCGTCTTACACCGTGCGGCACAGGCACTAGTACTG } \\
\text { ATGTCGTATACAGGGCTTTTGACATCTACAATGATAAAGAATAGACACCGCGAACCCATGCTT } \\
\text { TGGTTTTGCTAAATTCCTAAAAACTAATTGTTGTCGCTTCCAAG } \\
\text { AAAAGGACGAAGATGACAATTTAATTGATTCTTACTTTGTAGTT } \\
\text { AAGAGACACACTTTCTCTAACTAC }\end{array}$ \\
\hline
\end{tabular}

Table S8. Primer sets targeting N for SARS-CoV-2 microfluidic LAMP assay

\begin{tabular}{|c|c|}
\hline Project & Primer sequence (5'-3') \\
\hline N-F3 & GGCAGTCAAGCCTCTTCTC \\
\hline N-B3 & TTGCTCTCAAGCTGGTTCAA \\
\hline N-FIP & TTCCCCTACTGCTGCCTGGAGTTCCTCATCACGTAGTCGC \\
\hline N-BIP & TTCTCCTGCTAGAATGGCTGGCTCTGTCAAGCAGCAGCAAAG \\
\hline N-LB & AATGGCGGTGATGCTGCTCTT \\
\hline target & $\begin{array}{l}\text { GCGGCAGTCAAGCCTCTTCTCGTTCCTCATCACGTAGTCGCAA } \\
\text { CAGTTCAAGAAATTCAACTCCAGGCAGCAGTAGGGGAACTTCT } \\
\text { CCTGCTAGAATGGCTGGCAATGGCGGTGATGCTGCTCTTGCTT } \\
\text { TGCTGCTGCTTGACAGATTGAACCAGCTTGAGAGCAAAATGTC } \\
\text { TGGTAAAGGCCAACAACAACAAGGCCAAACTGTCACTAAGAA } \\
\text { ATCTGCTGCTGAGGCTTCTAAGAAGCCTCGGCAAAAACGTACT } \\
\text { GCCACTAAAGCATACAATGTAACACAAGCTTTCGGCAGACGT } \\
\text { GGTCCAGAACAAACCCAAGGAAATTTTGGGGACCAGGAACT }\end{array}$ \\
\hline
\end{tabular}

